\title{
PROCESOS DE DESCOMPOSICIÓN Y MACROINVERTEBRADOS ASOCIADOS EN UN RÍO ITALIANO
}

\author{
E.A. Fano', M. Zamorani² y J.G. Morgana ${ }^{2}$ \\ 1. Dipartamento Biologia Evolutiva. Via L. Borsari, 16. 44100 Ferrarn. Italia. \\ 2. Laboratorio di Ecologia, Dipartimento di Genetica e Biologia Molecolare «C. Darwin», \\ Università degli Studi di Roma «La Sapienza». Ple. Aldo Moro 5, 00185 Roma. Italia.
}

Palabras clave: leaf decomposition, stream, CPOM, FPOM. trophic groups

\begin{abstract}
DECOMPOSITION PROCESSES AND ASSOCIATED MACROINVERTEBRATES IN AN ITALIAN STREAM
\end{abstract}

The aim of this work is to analyse the decomposition trend of holm-oak (Quercus ilex L.) leaves in the Arrone Stream (Central Italy) during autumn and winter 1986-1987. Leaf processing rates were measured by confining leaf packs ( $8 \mathrm{~g}$ dry weight) in two zones, upstream and downstream, and calculating ash free dry weights remained after $7,14,28,42,70$ and 100 days of immersion. Our results show a faster decomposition process upstream $(\mathrm{k}=.0073)$ than downstream $(\mathrm{k}=.0046)$, and this was chiefly related with the different macrobenthic associations present at each sampling site; the shredder trophic group proportions at the two sampling sites differ significantly (chi-square test; $p>0.05$ ), and there was also significant differences between proportions of some of taxa of shredders (chi-square test; $\mathrm{p}<0.05$ ), showing that the association present at the first sampling site is able to utilize the detritus with greater effectiveness.

\section{INTRODUCCIÓN}

En los últimos años se ha evidenciado la importancia de la materia orgánica vegetal que entra en los ríos como fuente de energía para los macroinvertebrados fluviales (ANDERSON \& SEDELL, 1979; Cummins \& Klug, 1979; Short et al., 1980; DunCAN \& Brusven, 1985; Bird \& Kaushik, 1987; FAno et al., 1988 a, Cummins et al., 1989). La cantidad y las dimensiones de la materia orgánica cambian a lo largo del curso de agua, y por lo tanto la organización trófica de los macroinvertebrados se modifica en conformidad. En particular, según la teoría que considera un río como un continuo (VANNOTE et al., 1980), el metabolismo de un ecosistema lótico se basa sobre el detrito: CPOM («coarse particulate organic matter», diá-

Limnetica, 7: 61-69 (1991)

(C) Asociación Española de Limnología, Madrid. Spain metro $>1 \mathrm{~mm}$ ) en la zona de cabecera y FPOM («fine particulate organic matter», diámetro $<1$ $\mathrm{mm}$ ) en la zona de desembocadura, y las comunidades animales de cada tramo del río se caracterizan por el predominio de algunos grupos tróficos en relación al tipo principal de recurso nutricional. La relación existente entre recursos energéticos disponibles y predominio de algunos grupos tróficos es válida también en los tramos intermedios de ríos de grandes dimensiones, que recorren largos tramos en zonas colinares con escasa cobertura vegetacional, donde el metabolismo es autótrofo (Cummins, 1979).

El objetivo de este trabajo es el de evidenciar el modo en el que se descomponen con el tiempo las hojas de encina (Quercus ilex L.), que representan una fuente energética externa, en dos zonas, de cabecera y de desembocadura, del río Arrone (Italia). Tal proceso de descomposición ha sido estudiado en relación a la composición trófi- 
ca de los macroinvertebrados asociados y a las características físico-químicas y morfológicas del río en las zonas de muestreo.

\section{MATERIAL Y MÉTODOS}

\section{Área de estudio}

El río Arrone está situado en una área volcánica cerca de Roma. El origen del alveo fluvial es anterior al origen de la cadena volcánica de los Montes Sabatini (De Rita et al., 1983). Emisario del lago de Bracciano (fig. 1), desemboca en el Mar Tirreno después de haber recorrido $35 \mathrm{~km}$. El área de su cuenca es de $170 \mathrm{~km}^{2}$.

Fueron escogidas dos estaciones de muestreo: zona de cabecera, Galeria, y zona de desembocadura, Testa di Lepre. La distancia entre las dos localidades es de $16 \mathrm{~km}$. La zona de Galeria está a $8 \mathrm{~km}$ de distancia del lago de Bracciano; se eligió tal distancia porque fué considerada suficiente para evitar una influencia notable del lago sobre la estructura fluvial. Además, la estación de $\mathrm{Ga}$ leria corresponde por las características geomorfológicas y geográficas (río con bordes altos, flujo de agua interrumpido por barreras naturales, etc.) a una zona de cabecera. En la estación de Testa di Lepre, en cambio, el río Arrone fluye en modo regular en una zona llana. En las dos localidades de muestreo la profundidad del agua durante el período experimental fué parecida $(0,4-0,5 \mathrm{~m})$, mientras la anchura era mayor en Testa di Lepre $(4 \mathrm{~m})$ con respecto a Galeria $(3 \mathrm{~m})$. Comparaciones de las características físico-químicas y sedimentológicas de las zonas de muestreo están expuestas en la tabla 1 y en la figura 2 .

La vegetación ribereña es abundante en la zona de Galeria, de hecho abundan alisos (Alnus glutinosa), encinas (Quercus ilex), álamos (Populus nigra), sauces (Salix spp.), áceres (Acer spp.); por el contrario en Testa di Lepre existe una dominancia de vegetación herbácea, y sólo esporádicamente se encuentran algunos sauces (Salix spp.).

\section{Métodos de muestreo}

Treinta paquetes $\left(10 \mathrm{~cm} \times 10 \mathrm{~cm}, 0,5 \mathrm{~cm}^{2}\right.$ de malla) de hojas de Quercus ilex L. (8 $\mathrm{g}$ de peso seco) fueron sumergidos en el río, apoyados en el fondo, en cada zona experimental en el mes de octubre de 1986. Las hojas de encina fueron recogidas de árboles presentes en los bordes del cauce fluvial. En cada muestreo, realizado después de 7, 14, 28, 42, 70 y 100 días de inmersión. 5 paquetes de hojas fueron recogidos en cada zona experimental.

En las mismas fechas se examinaron las siguientes características físico-químicas: velocidad de corriente, temperatura, alcalinidad del agua (titulación acidométrica con HCI N/20), concentración de oxígeno disuelto (método Winkler). En referencia a las características sedimentológicas se consideraron la granulometría del sustrato $(0-3 \mathrm{~cm}$ de profundidad) y su contenido en materia orgánica. La granulometría fue calculada pasando el sedimento seco a través de cedazos con mallas decrecientes, y calculando el peso seco de las fracciones obtenidas. Además fueron calculados los valores de phi (phi $=-\log _{2}$ del diámetro en $\mathrm{mm}$ de las partículas) de la fracción dominante. El contenido de materia orgánica en el sustrato expreso

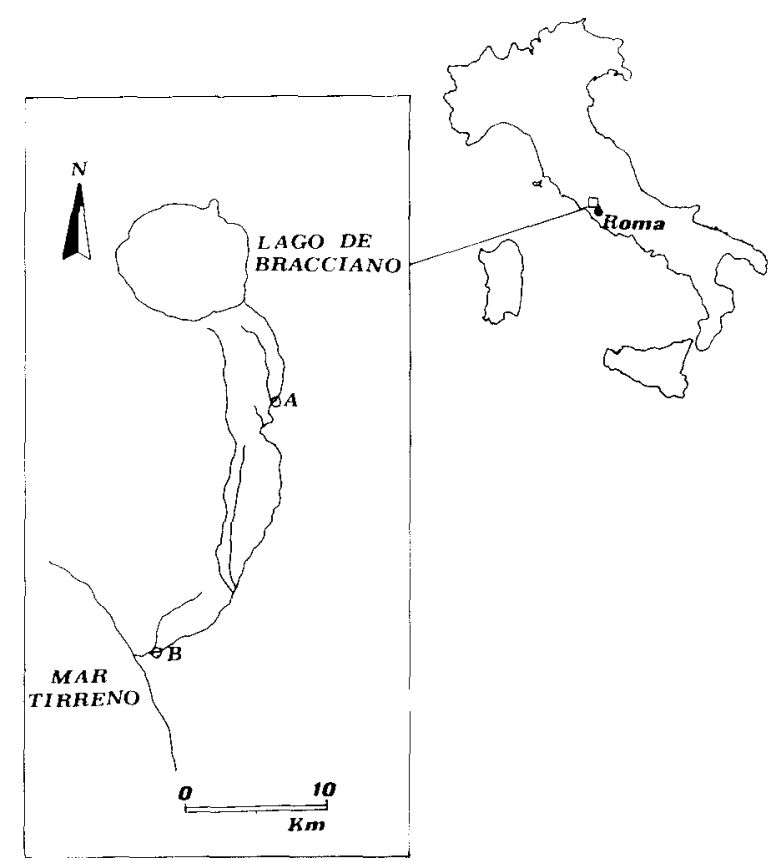

Figura 1.- Localización de las estaciones de muestreo en el río Arrone: $\mathrm{A}=$ Galeria, $\mathrm{B}=$ Testa di Lepre. Location of sampling sites in the Arrone stream: $A=$ Galeria, $\mathbf{B}=$ Testa di Lepre. 
Tabla 1.- Características químico-físicas del agua en las estaciones de muestreo. Se muestran además los valores de profundidad del río. $\mathrm{G}=$ Galeria, $\mathrm{T}=$ Testa di Lepre.

Water physico-chemical characteristics at the sampling sites. The stream depth values are also showed. $\mathrm{G}=\mathrm{Galeria}$. $\mathrm{T}=\mathrm{Testa}$ di Lepre.

\begin{tabular}{|c|c|c|c|c|c|c|}
\hline Fecha & Estación & $\begin{array}{c}\text { Temperarura } \\
\left({ }^{\circ} \mathrm{C}\right)\end{array}$ & $\begin{array}{c}\text { Oxigeno disuelto } \\
(p p m)\end{array}$ & $\begin{array}{l}\text { Alcalinidad } \\
\left.m g \mathrm{CaCO} . l^{\prime}\right)\end{array}$ & $\begin{array}{c}\text { Velocidad } \\
\text { de corriente } \\
(m, s-1)\end{array}$ & $\begin{array}{l}\text { Profundidad } \\
(m)\end{array}$ \\
\hline \multirow[t]{2}{*}{$27-09$} & $G$ & 18,0 & 13,0 & 223,5 & 0,42 & 0,50 \\
\hline & $T$ & 19,0 & 11,6 & 272,0 & 0,45 & 0.42 \\
\hline \multirow[t]{2}{*}{$04-10$} & $G$ & 17,5 & 8,8 & 227,5 & 0,27 & 0,42 \\
\hline & $T$ & 19,0 & 9,4 & 245,0 & 0,50 & 0,40 \\
\hline \multirow[t]{2}{*}{$11-10$} & $G$ & 18,0 & 9,9 & 250,0 & 0.44 & 0.42 \\
\hline & $T$ & 17.0 & 9,7 & 252,5 & 0.53 & 0,40 \\
\hline \multirow[t]{2}{*}{$25-10$} & $G$ & 13,5 & 11,9 & 237,5 & 0.25 & 0,40 \\
\hline & $T$ & 12,0 & 11,9 & 247,4 & 0.67 & 0,34 \\
\hline \multirow{2}{*}{$08-11$} & $G$ & 15,5 & 10.7 & 225,0 & 0.47 & 0,41 \\
\hline & $T$ & 12,0 & 11,9 & 260.0 & 0,38 & 0,34 \\
\hline \multirow[t]{2}{*}{$06 \cdot 12$} & $G$ & 11.5 & 10,4 & 237.5 & 0,27 & 0,42 \\
\hline & $T$ & 8.5 & 11.7 & 247,5 & 0,50 & 0,38 \\
\hline \multirow[t]{2}{*}{$05-01$} & $G$ & 9.5 & 9,9 & 250,0 & 0,54 & 0,58 \\
\hline & $T$ & 7,0 & 10,6 & 268,0 & 0.70 & 0,47 \\
\hline
\end{tabular}

como CPOM y FPOM fue calculado como peso seco (horno, $60{ }^{\circ} \mathrm{C}, 72 \mathrm{~h}$ ) sin cenizas (horno-mufla, $800{ }^{\circ} \mathrm{C}, 4 \mathrm{~h}$ ) de las fracciones mayores y menores de $1 \mathrm{~mm}$.

El proceso de descomposición de las hojas fué analizado calculando la pérdida de peso seco sin cenizas (AFDW, «ash free dry weight») durante el período experimental; para calcular tal biomasa, las hojas recogidas en cada muestreo fueron lavadas, secadas (horno, $60^{\circ} \mathrm{C}, 72 \mathrm{~h}$ ) e incineradas (horno-mufla, $800^{\circ} \mathrm{C}, 4 \mathrm{~h}$ ). Se calculó también el coeficiente $\mathrm{k}$ (Petersen \& Cummins, 1974): $\mathrm{k}=-\ln (\% \mathrm{R} / 100) \cdot \mathrm{t}^{\prime}$, donde $\mathrm{R}$ es el peso del material residual y $\mathrm{t}$ es el tiempo expresado en días.

Los macroinvertebrados encontrados en cada paquete fueron clasificados por taxón y por grupo trófico: trituradores, recolectores, raspadores, predadores (Merrit \& Cummins, 1978; Hawkins \& Sedell, 1981). Se calcularon las abundancias (frecuencias) de cada taxón y de cada grupo trófico, así como la constancia de cada taxón (c $=\mathrm{n}_{\mathrm{m}} / \mathrm{N}_{\mathrm{m}} ; \mathrm{n}_{\mathrm{m}}$ es el número de muestras en que se encuentra el taxón considerado y N,, es el número total de muestras). La afinidad en las composiciones de especies de las estaciones fue calculada usando el índice de similaridad entre dos muestras (SORENSEN, 1948). Además, se calculó el índice de diversidad trófica (HILDREw et al., 1984):
$\mathrm{D}=1 / \Sigma \mathrm{P}_{\mathrm{i}}^{2}$, considerando que $\mathrm{P}$, es el número de individuos (frecuencia relativa) de la categoría trófica i-esima.

Las asociaciones entre los taxones fueron evidenciadas mediante el coeficiente de Jaccard ( J ACCARD. 1908): $C,=n_{\mathrm{ab}} /\left(\mathrm{n}_{\mathrm{a}}+\mathrm{n}_{\mathrm{b}}+\mathrm{n}_{\mathrm{ab}}\right)$, consideran-

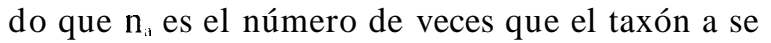
encuentra sólo en cada una de las muestras, $\mathrm{n}_{\mathrm{n}}$ es el número de veces que el taxón $b$ se encuentra en cada una de las muestras, y $n_{\text {ab }}$ es el número de veces en que los taxones $(a, b)$ se encuentran en cada una de las muestras. Se realizaron dendrogramas utilizando el algoritmo de la media ponderada (UPGMA) (SNeath \& Sokal, 1973) utilizando los valores del coeficiente de Jaccard para establecer un orden jerárquico en las agrupaciones de taxones. Para verificar la buena realización de los dendrogramas se calcularon los coeficientes de correlación cofenética obtenidos, comparando las matrices de valores originales (que fueron elaboradas para realizar los dendrogramas) y las matrices de valores cofenéticos.

\section{RESULTADOS}

Las características físico-químicas del agua y las características morfológicas de las estaciones de 
muestreo pueden verse en la tabla 1. Las variaciones de la temperatura y de la velocidad de corriente son mayores en Testa di Lepre que en Galeria. Los valores de la concentración de oxígeno disuelto y de la alcalinidad son parecidos en las dos localidades.

El análisis granulométrico del sustrato evidencia que las dos zonas de muestreo son diferentes. De hecho, en Galeria predomina la fracción comprendida entre 1 y $5 \mathrm{~mm}$, mientras que en Testa di Lepre predomina la fracción coniprendida entre 0,25 y $0,5 \mathrm{~mm}$ (fig. 2 ). Un predominio de CPOM $(63,71 \%$ por término medio $)$ se observa en el sustrato de Galeria, mientras que el FPOM $(82,18 \%$ por término medio) predomina en Testa di Lepre (fig. 3).

\section{GALERIA}

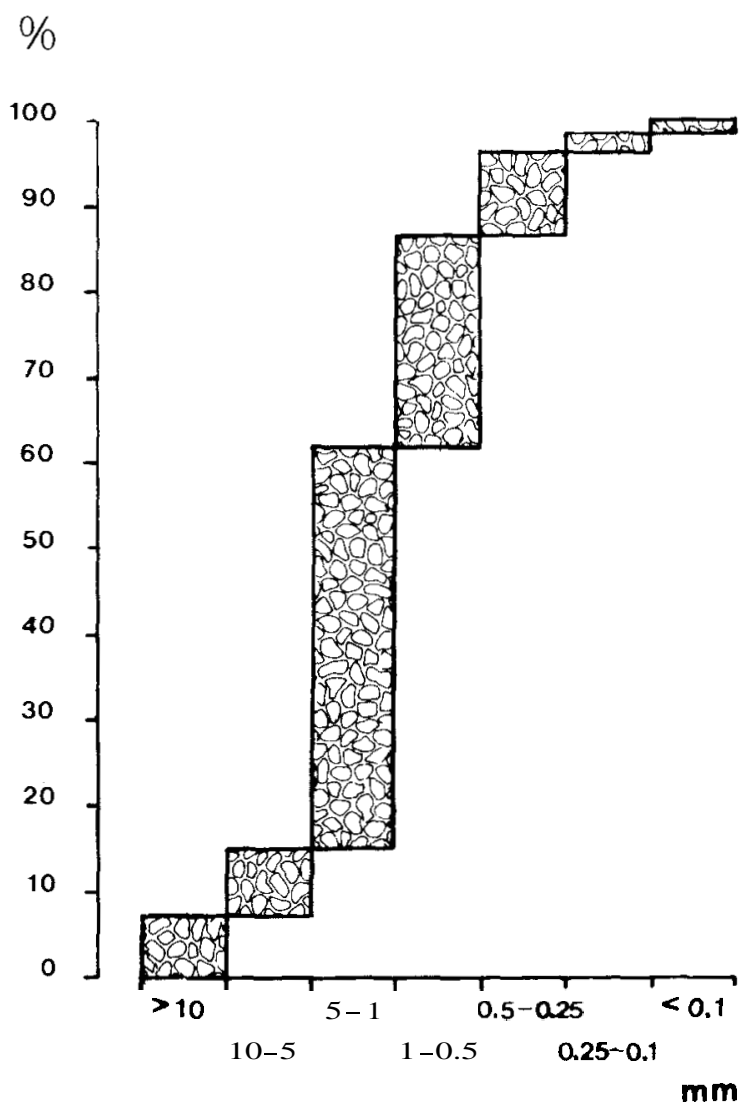

El proceso de descomposición de las hojas de Quercus ilex observado durante el período experimental resulta ser diferente en las dos estaciones de muestreo (fig. 4). En efecto, el proceso es más veloz en la zona de cabecera (queda el $48 \%$ de biomasa después de 100 días) con respecto a la zona de desembocadura (queda el $62 \%$ de biomasa en la misma fecha). El coeficiente de demolición más alto, relativo a la zona de Galeria $(\mathrm{k}=.0073)$, muestra que la descomposición de las hojas de Quercus ilex es moderada, mientras que en la zona de Testa di Lepre la descomposición es lenta $(\mathrm{k}=.0046)$.

El análisis de la colonización de los paquetes de hojas por parte de los macroinvertebrados evidencia que los taxones que presentan las mayores fre-

\section{TESTA DI LEPRE}

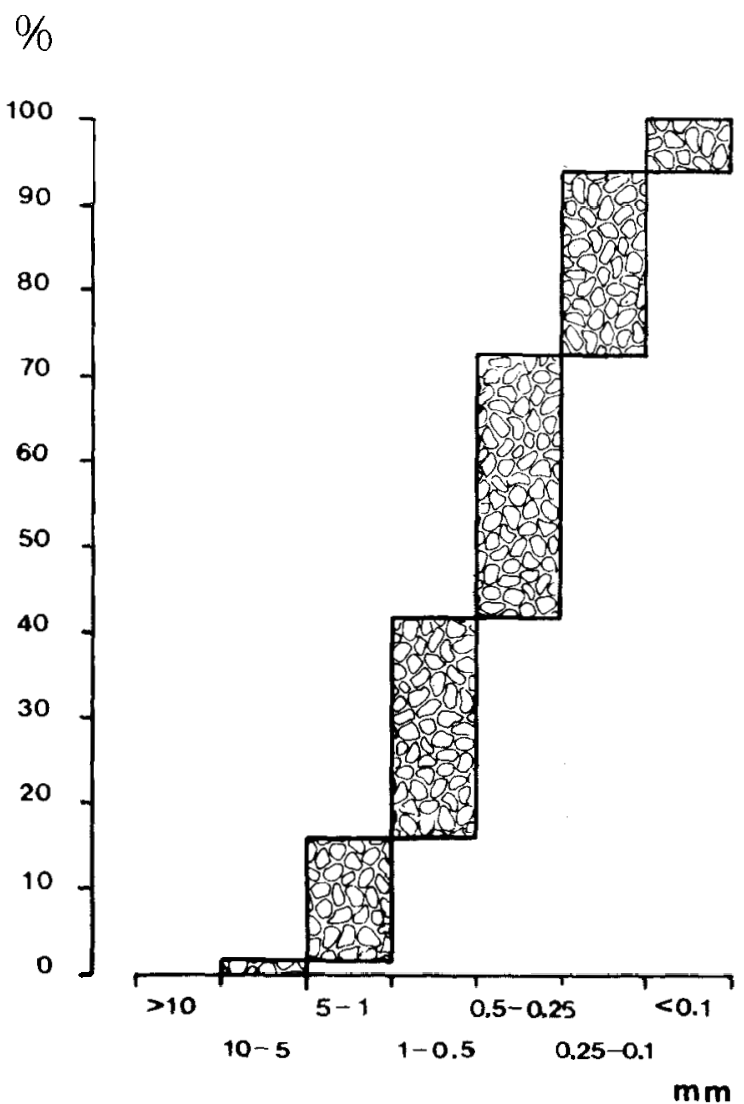

Figura 2.- Granulometría del sustrato recogido en las estaciones de muestreo. Se expresan como porcentajes las frecuencias de las diferentes fracciones.

Granulometry of the substratc collected at the sampling sites. The different fractions are expressed hy percentual frequencies. 

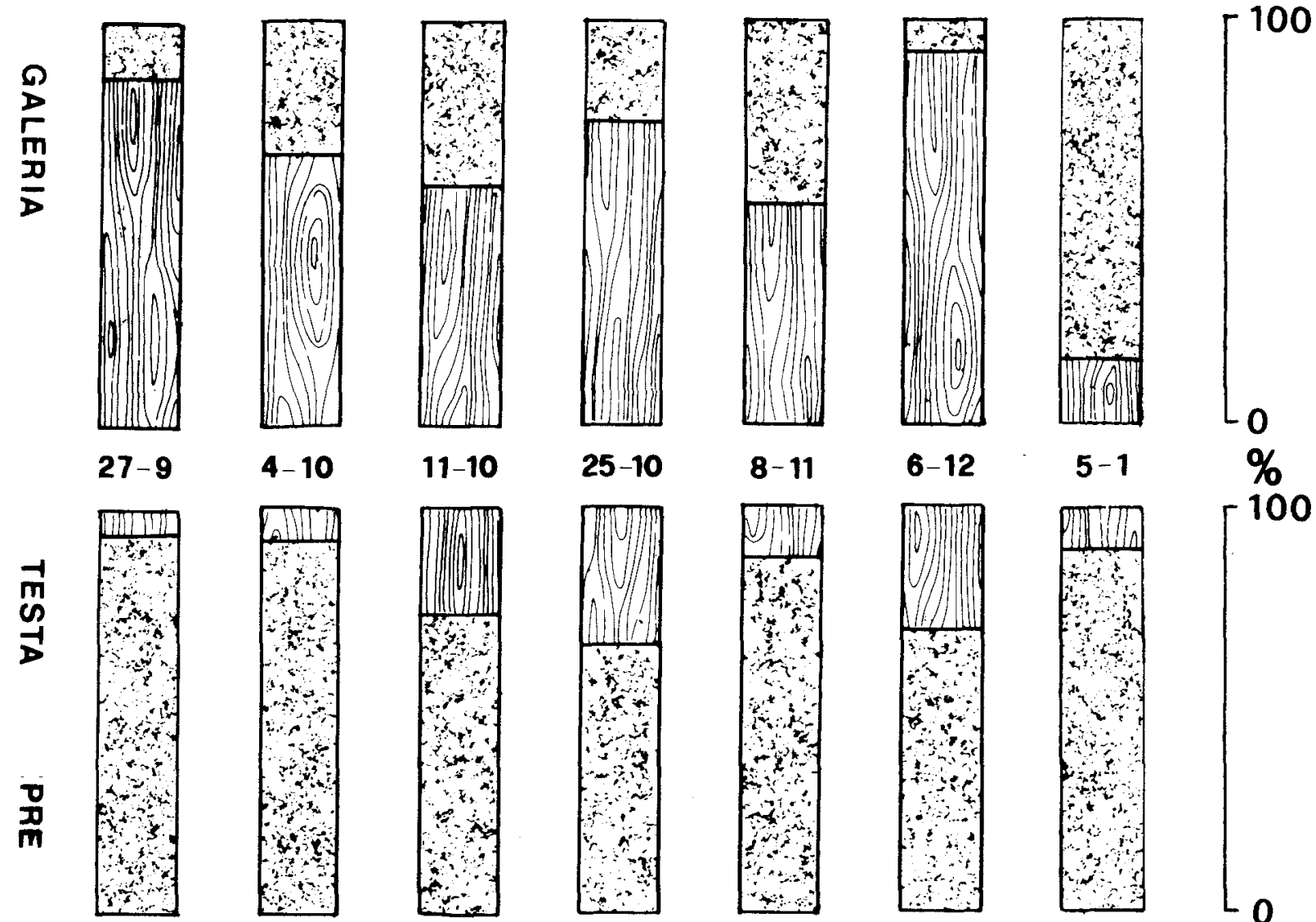

11-10
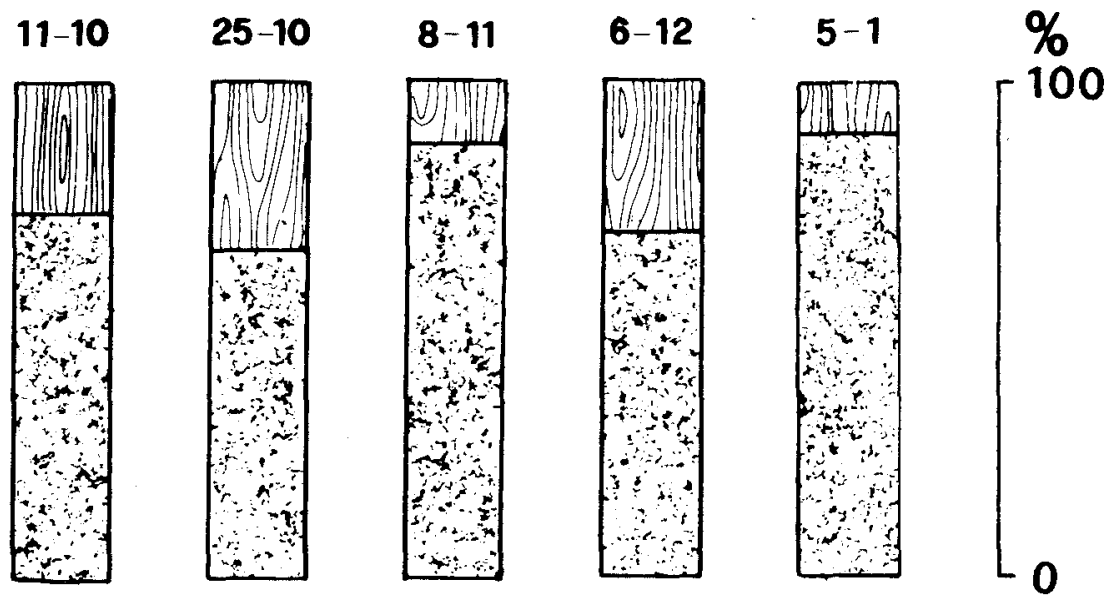

\section{$\%$ CPOM}

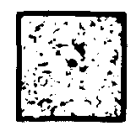

\section{$\%$ FPOM}

Figura 3.- Contenido (\%) de CPOM y FPOM en el sustrato recogido en cada estación en cada fecha de muestreo CPOM and FPOM content $(\%)$ in the substrate collected at each sampling site every sampling date.

cuencias (promedios) son los trituradores Proasellus coxalis, Echinogammarus sp., y Caenis macrura (tabla 2).

La comparación de las frecuencias medias (\%) de los grupos tróficos pone de manifiesto diferencias significativas (test $\mathrm{i}$-cuadrado; $\mathrm{p}<0,05$ ) en la colonización de los grupos trituradores y recolectores en las dos zonas de muestreo. El grupo de recolectores, consumidor de FPOM, predomina en la zona de desembocadura, y por el contrario, el grupo de trituradores resulta respresentado mayoritariamente en la zona de cabecera (tabla 2).
Los mayores valores de constancia los poseen los taxones Proasellus coxalis (en Galeria), Echinogammarus sp., Caenis macrura e Hydropsyche sp. (en Galeria y en Testa di Lepre).

Los promedios de los valores de los índices de diversidad trófica no son significativamente diferentes en las dos estaciones de muestreo (test $\mathrm{U}$ de Mann-Whitney; $\mathrm{p}>0,05)$. Tales promedios son: en Galeria, $\mathrm{D}=2,68$ (error $=0,289$ ), y en Testa di Lepre, $\mathrm{D}=2,31$ (error $=0,188$ ).

Los dendrogramas evidencian que en Galeria existe un mayor grado de asociación entre los 
Tabla 2.- Taxones encontrados en los paquetes de hojas de Quercus ilex colocados en las dos estaciones de muestreo $(G=$ Galeria, $\mathrm{T}=$ Testa di Lepre). Se muestran los valores de frecuencia (comparados con el test del qui-cuadrado, $\mathrm{p}<0.05$ ) y de constancia. Taxa found in the Quercus ilex leaf packs placed at two sampling sites $(\mathrm{G}=$ Galeria, $\mathrm{T}=$ Testa di Leprc). The frequency and constancy values are showed. The chi-square test $(\mathrm{p}<0.05)$ was achieved in order to compare the frequency values.

\begin{tabular}{|c|c|c|c|c|c|}
\hline & \multicolumn{2}{|c|}{ Frecuencia media $(\%)$} & \multirow[t]{2}{*}{ ji-cuadrado } & \multicolumn{2}{|c|}{ Constancia } \\
\hline & $G$ & $T$ & & $G$ & $I$ \\
\hline \multicolumn{6}{|l|}{ Trituradores } \\
\hline Proasellus coxalis & 33,56 & 0,06 & - & 0,80 & $0,0,1,3$ \\
\hline Echinogammaras sp & 13,75 & 41,90 & - & 0.57 & 0,83 \\
\hline Caenis macrura & 28.87 & 22,96 & ns & 0.90 & 0,83 \\
\hline Total & 76,18 & 64,92 & - & - & - \\
\hline \multicolumn{6}{|l|}{ Recolectores } \\
\hline \multicolumn{6}{|c|}{ Recolectores no filtradores } \\
\hline Ephemerella ignita & 0,54 & 1,07 & ns & 0,03 & 0,07 \\
\hline Chironomidae & 0.38 & 0.38 & ns & 0,03 & 0.27 \\
\hline Nematoda & 2.49 & 2,08 & ns & 0,33 & 0,27 \\
\hline Tubificidae & 0,54 & - & - & 0,07 & - \\
\hline \multicolumn{6}{|l|}{ Recolectores filtradores } \\
\hline Hydropsyche sp. & 4,84 & 8.61 & ns & 0,60 & 0,67 \\
\hline Wilhelmia sp. & 2,39 & 11,59 & - & 0,20 & 0,50 \\
\hline Total & 11.18 & 23.73 & - & & \\
\hline \multicolumn{6}{|l|}{ Predadores } \\
\hline Tricladida & 6.39 & - & - & 0,47 & \\
\hline Hirudinea 3.35 & 1,14 & ns & 0,40 & 0,13 & \\
\hline Hydracnidae & - & 0,77 & - & - & 0,13 \\
\hline Hydrophilidae & 1,33 & 3,73 & ns & 0.13 & 0,50 \\
\hline Total & 11,09 & 5,64 & ns & & \\
\hline \multicolumn{6}{|l|}{ Raspadores } \\
\hline Physa sp & 1.19 & 2,34 & ns & 0,10 & 0.30 \\
\hline Planorbis sp. & 0.40 & - & & 0.03 & - \\
\hline Total & 1.59 & 2.34 & $n s$ & - & - \\
\hline
\end{tabular}

taxones (fig. 5). Los taxones que presentan un buen grado de asociación en las dos localidades de muestreo son los que presentan mayor constancia. Los valores de los coeficientes de correlación cofenética son altos $(0,91714$ considerando el dendrograma de Galeria; 0,92969 considerando el dendrograma de Testa di Lepre), con lo que el nivel de realización de cada análisis es óptimo.

\section{DISCUSIÓN}

Tal como evidencian los resultados, resulta claro que las dos zonas de muestreo del río Arrone

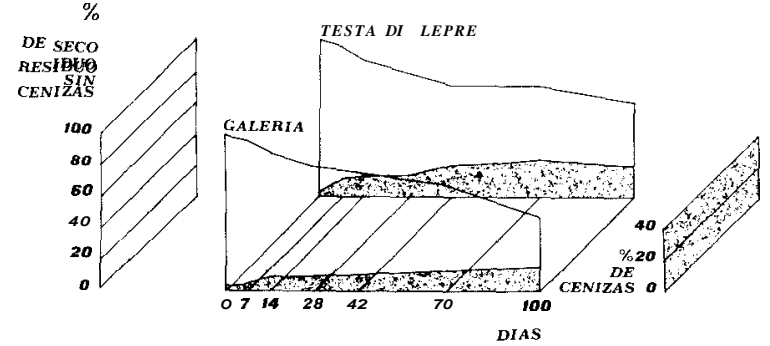

Figura 4.- Descomposiciún de las hojas de Quercus ilex en las dos estaciones de muestreo del río Arrone: pérdida de peso seco sin cenizas $(\%)$ y contenido de cenizas $(\%)$.

Processing of Quercus ilex leaves at the sampling sites of the Arrone stream: ash free dry weight $(\%)$ and ashes content $(\%)$. 


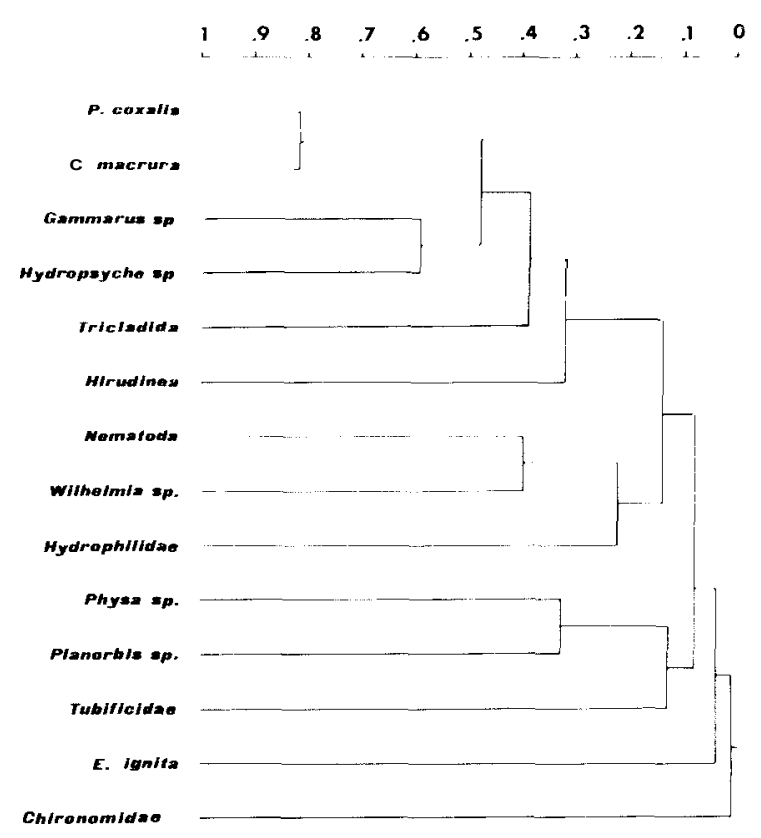

Figura 5.- Agrupaciones de taxones Taxa clustering.

son diferentes por lo oque se refiere a los factores físico-químicos y sedimentológicos (tabla 1, figs. 2, 3). En particular, los parámetros que mayormente distinguen las dos estaciones son: a) Los valores de temperatura que resultan casi siempre más elevados en la estación de Galeria. Este hecho probablemente se pone en relación con los intercambios con el ambiente circundante, que son mayores en la zona de Testa di Lepre. De hecho, en esta estación, la interfacie aire-agua es más amplia, y por lo tanto las variaciones de la temperatura del agua dependen más de las variaciones térmicas del ambiente aéreo. Además, la escasa cobertura de vegetación de la zona de Testa di Lepre permite un mayor grado de intercambio con el ambiente; b) la granulometría del sustrato. De hecho, en la zona de cabecera la fracción más común es la que resulta comprendida entre 1 y $5 \mathrm{~mm}$, mientras que en Testa di Lepre es aquella comprendida entre 0,25 y $0,50 \mathrm{~mm}$. Por tanto, en la estación de Galeria predomina la grava fina (phi $=-1)$, mientras que en la estación de Testa di Lepre predomina la arena media (phi $=2$ ) (WENTWORTH, 1922); c) La importancia relativa de las fracciones de CPOM y FPOM en el sustrato. Los valores en las dos estaciones son variables en el tiempo, y se observó un 63,71\% de CPOM en Galeria y un $17,82 \%$ de CPOM en Testa di Lepre. Estos datos evidencian que en la zona de cabecera predomina la materia orgánica particulada gruesa, mientras que en la zona de desembocadura predomina la materia orgánica particulada fina.

Todas estas características concuerdan con 10 que ha sido propuesto sobre los gradientes físicoquímicos y sedimentológicos de los ecosistemas lóticos (Cummins, 1979; Vannote et al., 1980; MinsHALL et al., 1983).

La velocidad de descomposición de las hojas de Quercus ilex fué también diferente en las dos zonas de muestreo. En efecto, tal velocidad fué mayor en Galeria $(\mathrm{k}=.0073)$ que en Testa di Lepre $(\mathrm{k}=$,0048). Esta diferencia en la velocidad de descomposición puede estar relacionada con los distintos valores de temperatura evidenciados anteriormente, ya que se observa una velocidad mayor en la zona con temperatura más alta. De hecho ha sido observada una relación directa entre velocidad de descomposición y temperatura (IVER- 
SEN, 1975; PAUl et al. 1978; Short et al. 1984), ya que la temperatura influye sobre :a actividad de los microorganismos y de los invertebrados implicados en los procesos de descomposición de la materia orgánica (Suberkropp \& Klug, 1976; AN derson \& Sedell, 1979; Cummins \& Klug, 1979).

En nuestro trabajo se analizaron las asociaciones de macroinvertebrados que colonizaban los paquetes de hojas. No se observaron diferencias en el tipo de colonización de cada paquete en cada estación. Este resultado se debe al tipo de muest $r$ e utilizado y también al medio de las zonas estudiadas que era homogéneo, ya que no representaba ull mosaico de microambientes (MuÑozet al.. 1986); por este último motivo suponemos que las especies animales encontradas en los paquetes correspondían a las del área circundante.

En referencia a las asociaciones de macroinvertebrados que colonizaron los paquetes, de hojas en las dos estaciones de muestreo, no se observaron grandes diferencias en la composición de los taxones. De hecho, el índice de similaridad de Sörensen resultó alto $(\mathrm{S}=.846)$. Por el contrario, diferencias significativas se observaron entre las abundancias (frecuencias medias expresas como porcentajes) de algunos taxones y de algunos grupos tróficos. En efecto, el grupo de los trituradores resultó ser significativamente mayor representado en Galeria; y también los taxones que forman el mismo grupo fueron presentes de diferente manera en las dos estaciones: en Galeria se observó una dominancia del isópodo Proasellus coxalis, y en cambio en Testa di Lepre predominó el anfípodo Echinogammarus sp. La mayor abundancia del grupo de los trituradores en la zona de cabecera se pone en relación con la gran cantidad de CPOM en el sedimento; así como la abundancia significativamente mayor del grupo de los recolectores + filtradores en Testa di Lepre se relaciona con la mayor importancia de FPOM en tal estación. siendo respectivamente estos grupos tróficos los específicos de tales categorías de detrito (Anderson \& Sedell, 1979; Cummins \& KLUG, 1979).

Las abundancias de los depredadores no son significativamente diferentes en las dos estaciones. Se puede observar que los tricladidos representan la mitad de los depredadores en Galeria, mientras que en Testa di Lepre están ausentes (tabla 2). Este dato se puede relacionar con la gran abun- dancia de Asélidos presentes en Galeria. En efecto. se ha visto que los miembros del grupo "ricladidae son los depredadores más comunes de los Asélidos (De SiLVA, 1976).

La baja frecuencia del grupo de los rascadores sc debe a dos razones: al ambiente estudiado que es principalmente heterótrofo (Minshall et al., 1983; FAno et al.. 1988b), y al método de muestreo utilizado. ya que los paquetes de hojas representan sin duda un microhabitat favorable para los organismos de la cadena alimentaria implicados en el procesamiento de la materia orgánica (FANO \& Rossi. 1982).

Si se relaciona la estructura de las comunidades animales con la velocidad de descomposición de las hojas, se puede explicar la mayor velocidad de descomposición en la zona de cabecera con la mayor frecuencia del grupo de los trituradores y sobretodo con la asociación entre Proasellus coxalis y Caenis macrura, que de hecho parece ser más hábil en descomponer las hojas que la asociación entre Echinogammarus sp. y Caenis macrura presente en cambio en Testa di Lepre. Como ha sido evidenciado en otros trabajos (КНокт et al., 1980; Benfield \& Webster, 1985, Pearson et al., 1989) el grupo de trituradores tiene gran importancia en relación con la velocidad de descomposición. En nuestro estudio, sólo la mayor abundancia del grupo de trituradores en Galeria no es suficiente para garantizar una descomposición más veloz, ya que no presenta una correlación significativa entre el nivel diario de descomposición y la entidad de colonización por parte de los trituradores, sino que resulta fundamental también la especie más abundante de tal grupo trófico, así como su eficiencia en la utilización del detrito, que resulta variable según el tipo de detrito y el tipo de colonización microbiana (ARSUFFI \& SUbERKRopp, 1984; FANO et al., 1982).

La rápida descomposición observada en Galeria se puede relacionar con la mayor diversidad taxonómica y funcional encontrada en esta estación. En efecto, los valores de diversidad trófica, si bien no sean significativamente diferentes, son mayores en Galeria con respecto a Testa di Lepre; esto concuerda con los dendrogramas relativos a las dos asociaciones, ya que en Galeria resulta más complejo que en Testa di Lepre. En Galeria, la mayor asociación entre los taxones corresponde a una actividad animal mayor, que 
aumenta la actividad metabólica del sistema y la velocidad de descomposición (Bl:RrIt, 1976).

En conclusión, la descomposición que viene influenciada por los macroinvertebrados depende de: 1) la eficiencia de descomposición del taxon o de los taxones dominantes del grupo de trituradores; 2) la complejidad de la asociación de los macroinvertebrados. En nuestro estudio esto se concluye en una descomposición más activa en la estación de Galeria.

\section{Bibliografía}

Anderson, N.H. \& J.R. Sedill, 1979. Detritus processing. by macroinvertebrates in stream systems. Ann. Rev. Entomol., 24: 351-377.

ARSUFF, T.L. \& K. SUBERKROPP, 1984. Leaf processing capabilities of aquatic hyphomycetes: interspecific differences and influence on shredder feeding preferences. Oikos, 42: 144-154.

Benfield, E.F. \& J.R. WEBster, 1985. Shredders abundance and leaf breakdown in an Appalachian mountain stream. Freswater Biol., 15: 113-120.

BERRIE, A.D., 1976. Detritus, micro-organisms and animals in fresh water. In: (eds.), J.M. ANDERSON \& A. MACFADYEN. The Role of Terrestial and Aquatic Organisms in Decomposition Processes, 323-338 pp. Blackwell Scientific Publications, Oxford.

Bird, G.A. \& N.K. Kaushik, 1987. Processing of maple leaf, grass and fern packs and their colonization by invertebrates in a stream. J. Freshwater Ecol., 4 (2): 177-189.

Cummins, K.W., 1979. The natural stream ecosystem. In: WARD, J.V. \& J.A. STANFORD (eds.), The Ecology of regulated streams, 7-24 págs. Plenum press, New York and London.

Cummins, K.W. \& M.J. Klug, 1979. Feeding ecology of stream invertebrates. Ann. Rev. Ecol. Syst., 10: 147-172

Cummins, K.W., M.A. Wilzback, D.M. Gates, J.B. Perky \& W.B. Taliaferro, 1989. Shredders and Riparian vegetation. Bioscience, 39 (1): 24-30.

De Rita, D., R. Funicello, U. Rossi \& A. Sposato, 1983. Structure and evolution of the Sacrofano-Baccano Caldera, Sabatini volcanic complex, Rome. $J$, of Vulc. and Geoth. Res., 17: 219-236.

DE Silva, P.K., 1976. The factors affecting the feeding of Dendrocoelum lacteum [Müller] (Turbellaria, Tricladida) on Asellus aquaticus (L.) (Crustacea, Isopoda). Arch. Hydrobiol., 77 (3): 347-374.

DUNCAN, W.F.A. \& M.A. BrusVEN, 1985. Energy dynamics of three low-order southeast Alaska streams: allochthonous processes. J. Freswater Ecol., 3 (2): 233-248.

FANO, E.A. \& L. Rossi, 1982. Capacità portante del detrito vegetale in un corso d'acqua dell'Italia centrale. Boll. Zool, 49: 71 .

Fano, E.A., L. Rossi \& A. Basset, 1982. Role of fungi in the diet of three benthic detritivorous species. Boll. Zool., 49: $29-34$
Fano, E.A. I. Castilinuovo, M. Colangilo, E. Marrhi:1 il, J.G. Morgana, G. Puppl \& M. Zamorani, 1988a. Dccomposition process of Quercus ilex leaves in Mignone River (lialy). Boll. Mus. St. Nat. Lunigiana, 6-7: 127-131.

Fano, E.A., L. Castilinuovo, E. Marchetti, J.G. MorciaNA \& M. ZAMORANI, 1988b. An approach to the eriver continuum» theory. Boll. Mus. St. Nat. Lunigiana, 6-7: $325-328$.

MAWKINS, C.P.\& J.R. SIDHI. 1981. Longitudinal and seasonal changes in functional organization of macroinvertebrate communities in four Oregon streams. Ecology, 62 (2): $387-397$.

HIIDRI,W, A.G., C.R. Townstend \& J. Francis, 1984. Community structure in some southern English streams: the influence of species interactions. Freswater Biol. 14: 297 310.

IVIRSIN, T.M., 1975. Disappearance of autumn shed beech leaves placed in batgs in small streams. Verh. Internat. Verein. Limnol., 19: 1687-1692.

JAC'CARD), P., 1908. Nouvelles recherches sur la distribution florale. Bull. Soc, Vaud. Sci. Nat., 44: 223-270.

Mirrit, R.W. \& K.W. Cummins. 1978. An introduction to the aquatic insects of North America. In: MERRIT, R.W. \& K.W. Cummins (eds.), págs. 1-441. Kendall-Hunt, Dubuque. Iowa

Minshall, G.W., R.C. Petersen, K.W. Cummins, T.l. Bott, J.R. Sedell, C.E. Cushing \& R.L. Vannote, 1983. Interbiome comparison of stream ecosystem dynamics. Ecol. Monogr., 53: 1-25.

Muñoz, I., N. Prat, X. Millet, E. Martínez. Ansemil. 1986. Heterogeneidad espacial en la distribución de los macroinvertebrados a lo largo de un transecto en el río Llobregat (Barcelona, España). Limnética, 2: 135-145.

Paul, R.W., E.F. Benfield \& J. Jr. Cairns, 1978. Effects of thermal discharges on leaf decomposition in a river ecosystem. Verh. Internat. Verein. Limnol., 20: 1759-1766.

Pearson, R.G., R.K. Tobin, R.E.W. Smith \& L.J. Benson, 1989. Standing crop and processing of rainforest litter in a tropical Australian stream. Arch. Hydrobiol. 115 (4): 481-498.

Pitersen, R.C. \& K.W. Cummins, 1974, Leaf processing in a woodland stream. Freshwater biology, 4: 343-368.

SHORT, R.A. S.P. CANTON \& J.V. WARD, 1980. Detrital processing and associated macroinvertebrates in a Colorado Mountain stream. Ecology, 61 (4): 727-732.

Short, R.A., S.L. Smith, D.W. Guthrie \& J.A. Stanford, 1984. Leaf litter processing rates in four Texas streams. $J$. Freshwater Ecol., 2: 469-473.

SNEATH, P.H.A. \& R.R. SOKal, 1973. Numerical taxonomy. W.H. Freeman and Company, San Francisco, 573 pp.

SORENSEN, T., 1948. A method of establishing groups of equal amplitude in plant society based on similarity of species content. K. Danske Vidensk. Selsk., 5: 1-34.

SubERkropP, K. \& M.J. Kiug, 1976. Fungi and bacteria associated with leaves during processing in a woodland stream. Ecology, 57: 707-719.

Vannote, R.L., G.W. Minshali, K.W. Cummins, J.R. Se. DELL \& C.E. CuSHING, 1980.T The River Continuum Concept. Can. J. Fish. Aquat. Sci., 37: 130-137.

Wentworth, C.K., 1922. A scale of grade and class terms for clastic sediments. J. Geology, 30: 377-392. 